\title{
Facial Nerve Neuroma Management
}

\author{
Peter C. Weber, M.D. and J. David Osguthorpe, M.D.
}

\begin{abstract}
Three facial nerve neuromas were identified in the academic year 19941995. Each case illustrates different management dilemmas. One patient with a grade III facial nerve palsy had a small geniculate ganglion neuroma with the dilemma of decompression versus resection clear nerve section margins. The second patient underwent facial neuroma resection with cable graft reconstruction, but the permanent sections were positive. The last patient had a massive neuroma in which grafting versus other facial reconstructive options were considered. These three cases illustrate some of the major controversies in facial nerve neuroma management. We discuss our decision-making plan and report our results.
\end{abstract}

Like acoustic neuromas, facial neuromas are benign, slow-growing neoplasms that can occur anywhere along the course of the facial nerve. The actual incidence in the human population is unknown, but since the first report by Schmidt in $1930^{1}$ less than 400 cases have been reported ${ }^{2}$ in the literature.

Symptoms that arouse suspicion of a facial neuroma include facial paresis/paralysis, facial pain, facial spasm, hearing loss, or a combination of the above ${ }^{3,4}$ The most common presentation of a facial neuroma is gradual progression of facial palsy to complete paralysis $^{2-7}$. Bell's palsy, facial paralysis of acute onset, occurs in 10 to $15 \%$ of facial neuromas. ${ }^{2-5}$ This incidence rate has led to the recommendation by some that magnetic resonance imaging (MRI) with gadolinium be performed at some point in all patients with suspected Bell's palsy. ${ }^{5,8} \mathrm{~A}$ more conservative approach is to image only those patients in whom full recovery does not occur, recurrent paralysis occurs, or hemifacial spasm occurs. ${ }^{2,8}$ Other symptoms such as hearing loss (either conductive or sensorineural), vestibulopathies, dysgeusia (altered taste), decreased salivary flow, facial pain, or tearing with mastication can occur depending on the location of the neuroma. ${ }^{3,5,7}$

Radiologic tests are essential, not only to assist in the diagnosis, but also to develop an operative plan. Like acoustic neuromas, MRI with gadolinium allows visualization of the presumed extent of the facial neuroma. Computed tomography (CT) of the temporal bone is often useful in assessing the extent of bony erosion. This is extremely helpful in preoperative counseling and assessing the possible increased chance of postoperative vertigo or sensorineural hearing loss. Even with sophisticated imaging techniques, a facial neuroma can be confused with an acoustic neuroma. ${ }^{4} \mathrm{An}$ audiogram is essential to document any existing conductive or sensorineural hearing loss, although such may or may not be associated with the facial nerve neuroma. Electronystagmography, auditory brainstem response, and topographic testing do not enhance the information needed in the evaluation of facial neuromas or paralysis. 3.7

The purpose of this study is to highlight treatment options for facial neuromas. We demonstrate that each patient and neuroma present their own dilemma to treatment and that there is not always one best treatment option.

\section{CASE REPORT 1}

A 42-year-old white female was referred with a right facial nerve neuroma. She initially presented with a 9-month history of mild right cheek swelling, associated with pricking sensations. A CT scan demonstrated a large cystic intraparotid mass. At the time of parotidectomy, a partially cystic mass was found to be adherent to the medial surface of the facial nerve. This mass was removed by subtotal parotidectomy with gross facial nerve preservation, but with what was thought to be 
likely residue at the stylomastoid foramen. The patient did well postoperatively, except for a pronounced right facial weakness that required eye care at night. Patient had no personal or family history of tumors. Pathology from the parotidectomy was consistent with a right facial nerve schwannoma.

On physical examination in our office, the patient was noted to have a substantial House-Brackmann grade $\mathrm{V}$, right-sided facial weakness ${ }^{9}$ with a $2.5-\mathrm{mm}$ gap between the upper and lower lids while attempting to close the right eye. A Bell's phenomenon was present. There was essentially no movement in the lower branches of the right facial nerve. She had expected numbness over the superior branches of the cervical plexus, and the parotidectomy incision was well healed. An MRI with contrast was obtained that demonstrated enhancement along the right facial nerve to the junction between the horizontal and vertical segments with fallopian canal enlargement identified in the lower half of the vertical segment (Fig. 1).

The patient was taken to the operating room, where she underwent completion right total parotidectomy with facial nerve sacrifice. A mastoidectomy was performed with removal of facial nerve in the vertical segment. Frozen section on the proximal stump was interpreted as being negative for neuroma. Unfortunately, on permanent section, the margin was identified to be positive. The dilemma of close observation of the patient versus re-excision was discussed. The patient was brought back to the operating room for removal of the facial nerve to the geniculate ganglion. The permanent sections of the proximal margin were thereafter negative. She has been followed for approximately 1 year without evidence of

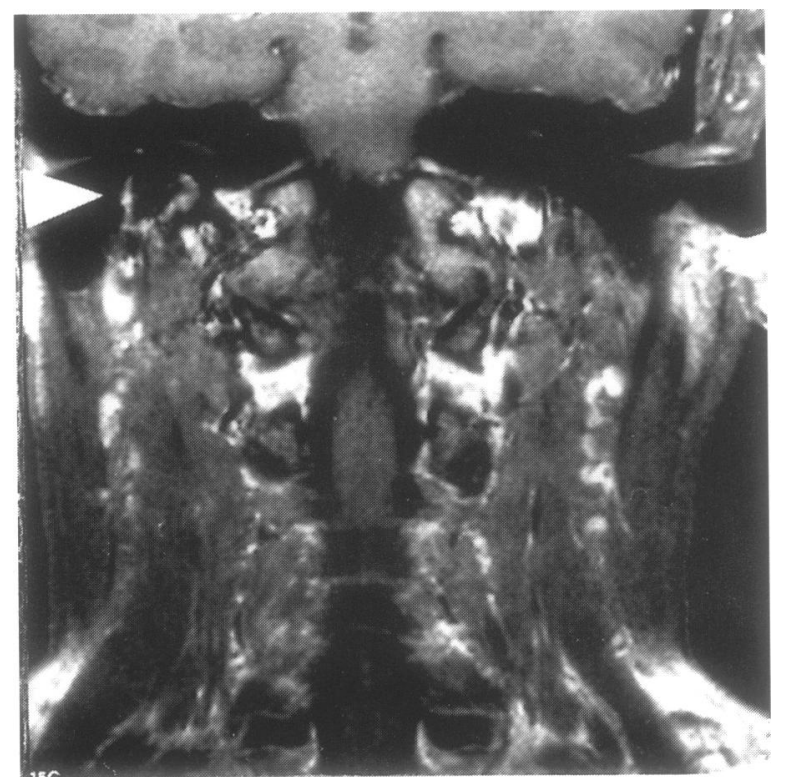

Figure 1. Coronal MRI with contrast demonstrating enhancement of the right facial nerve. recurrence, and facial nerve function has now returned to grade IV.

The dilemma of a positive margin on permanent pathologic review after frozen sections were interpreted as negative, and a cable graft had been placed. Even though this tumor is benign and slow growing, not resecting it completely may lead to further complications. Residual tumor may impede the regenerating axons associated with cable grafting, and certainly as the tumor increases in size it will again produce facial paresis. Reexcision at this time, when facial paresis or other symptoms dictate, may result in facial function that is substantially worse than what would have otherwise been expected. The risks of hearing loss or vestibulopathies with continued growth are also concerns. Therefore, we would recommend performing prompt re-excision when learning of positive margins. The uninvolved cable graft anastomosis may be left undisturbed, and re-anastomosis may occur at the re-excised site if the graft is of adequate length. If the graft is not of adequate length, a second, shorter cable graft may be spliced in between the original graft and the facial nerve or an entirely new cable graft can be harvested.

\section{CASE REPORT 2}

A 40-year-old white female presented with an external auditory canal/parotid and cerebellopontine angle (CPA) mass. She had initially been evaluated 10 years prior by another otolaryngologist for a parotid mass, and 10 years ago an initial biopsy was consistent with neuroma. Although surgery was recommended, the patient deferred. Two years after her initial diagnosis, she developed a grade VI facial nerve paralysis, and she subsequently developed a total hearing loss.

She now presented with a mass protruding out of her external auditory canal and a large parotid mass. She continued to have complaints of facial paralysis and hearing loss, although she denied other cranial nerve signs such as voice change, dysphagia, odynophagia, vertigo, disequilibrium, or otalgia.

On examination the right ear had a large polypoidtype mass extruding from the external auditory canal, with squamous debris trapped around the mass. She had a House-Brackman Grade VI facial nerve paralysis, ${ }^{9}$ but the remainder of her cranial nerves were grossly intact. An audiogram demonstrated profound sensorineural hearing loss on the right ear. Fine-needle biopsy obtained from the parotid and the external auditory canal mass and pathology were consistent with neuroma. CT (Fig. 2) and MRI scans (Fig. 3 and 4) demonstrated a mass involving the right temporal bone, right parotid gland, internal auditory canal, and CPA cistern that was very suggestive for a facial nerve schwannoma.

The patient was brought to the operating room, where a total parotidectomy with facial nerve sacrifice 


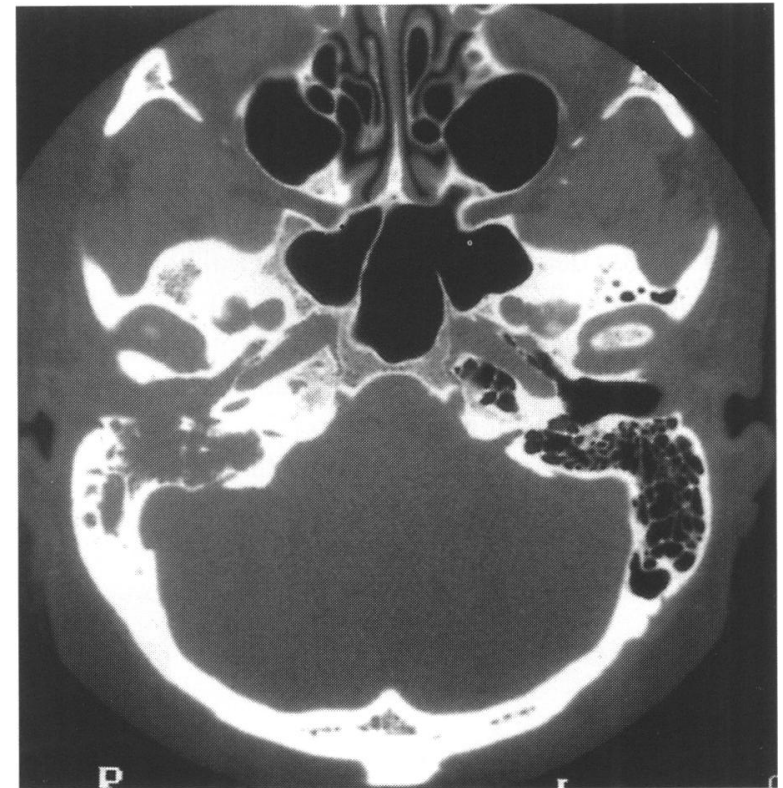

Figure 2. CT scan demonstrating the massive destruction of the right temporal bone.

was performed along with complete tumor removal through translabyrinthine and transcochlear approaches. This necessitated removal of the seventh and eighth nerve complexes at the brainstem. An upper lid gold weight was also inserted. The patient is 18 months postresection and has not developed any evidence of recurrence. Since cable grafting could not be performed, she has since undergone a right temporalis muscle transfer, right brow lift, right lateral tarsal strip, and a "fill in" procedure of her temporal fossa.

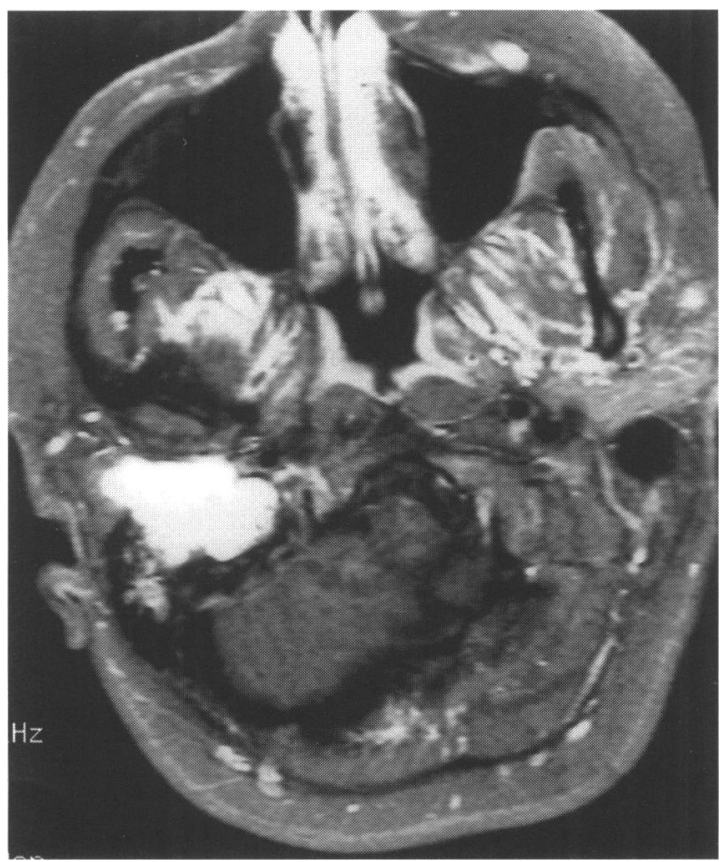

Figure 3. Axial enhanced MRI demonstrating the CPA, external auditory canal, and parotid mass.

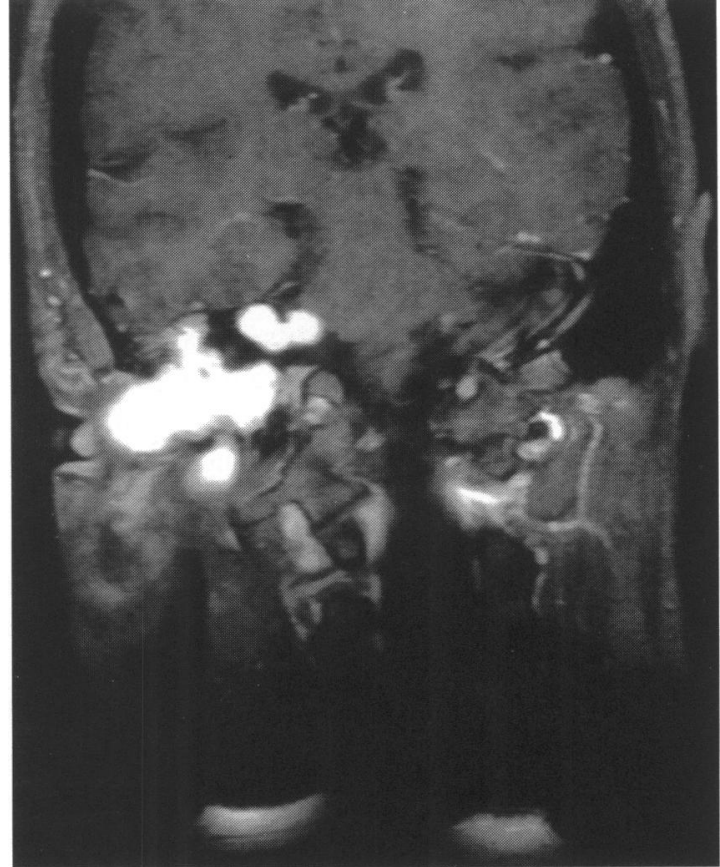

Figure 4. Enhanced coronal MRI with gadolinium demonstrating the large facial neuroma.

This case illustrates that not all facial nerve neuromas may be resected and repaired with cable grafting. When a large tumor is removed and an adequate proximal stump or distal branches are not suitable for cable grafting, alternative reanimation procedures are necessary. One such procedure is insertion of a gold weight into the upper eyelid to assist in eye closure and to avoid keratitis or conjunctivitis. The patient remained with significant complaints about the inability to use her orbicularis oris. Thus, the patient also underwent a right temporalis muscle transfer, right brow lift, and a lateral tarsal strip that has improved this patient's appearance considerably.

\section{CASE REPORT 3}

A 50-year-old white female presented with a rightsided facial nerve paresis of 2 years duration. She stated that originally she was diagnosed with Bell's palsy and treated with steroids but had never gotten complete return of facial function. Recently, she had thought that the facial nerve paresis may have gotten slightly worse, with symptoms that included numbness over the right side of the tongue and loss of taste sensation. She also at times felt some muscle twitching of her right face. She denied any hearing loss, tinnitus, vertigo, or disequilibrium. She complained of a dry right eye and was using lacrilube at night.

On examination, she was thought to have a HouseBrackmann grade II to III facial nerve paresis. ${ }^{9}$ The remaining cranial nerves were intact. Cerebellar testing and other office vestibular tests were normal. There did 
not appear to be any middle ear mass, and an audiogram confirmed normal hearing. A MRI scan was performed that demonstrated an enhancing mass (gadolinium) measuring approximately $5 \times 8 \times 10 \mathrm{~mm}$ centered on the right geniculate ganglion and most likely representing a seventh nerve neuroma (Fig. 5). It appeared as if the labyrinthine and proximal tympanic segments of the right seventh nerve were also involved. The patient was counseled on the risks and benefits of excision with cable grafting. The patient did not want to lose any more of her current facial nerve function and did not want to take the chance of the nerve function not returning to its present status. Thus, decompression of the nerve/neuroma was also discussed. The patient underwent decompression via a middle cranial fossa approach with the understanding that future resection and grafting would be needed and that she could lose her hearing or balance function if there was bony erosion into the cochlear vestibule. At the time of surgery, a small enlargement of the nerve was identified and decompressed. She has been followed for 1 year, and her first year MRI scan showed only minimal enlargement and she remains at a grade II to III paresis. She will continue with yearly scans unless she decides to have the tumor removed.

The last patient's case illustrates a controversy that is uncommon. Although surgical excision is the treatment of choice for facial nerve neuromas, it has been suggested that decompression may be an option in some cases. ${ }^{4,7}$ Our patient's facial nerve function was a grade II to III on the House-Brackman scale, and she had a relatively small neuroma. We discussed surgical excision with cable grafting, but, knowing that her facial nerve function would not

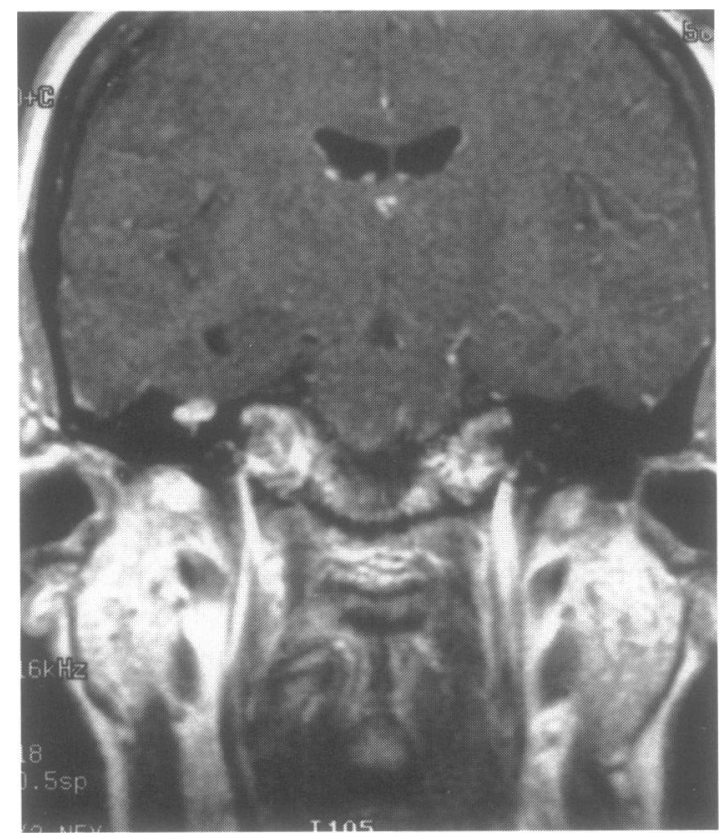

Figure 5. Enhanced MRI demonstrating the small facial neuroma and the geniculate ganglion. be any better than it currently was and that it would be worse initially and possibly worse long term, she did not want to undergo excision and grafting nor did she want a biopsy. We discussed the risks of hearing loss, vestibulopathies, and other cranial nerve abnormalities should this tumor progress in size. Since this was a small tumor, we did discuss decompression, which would hopefully allow her to enjoy facial function at her present grade for a somewhat longer period of time, hopefully many years. One year after decompression via middle cranial fossa approach, she remains at a grade II to III paresis, but is having increasing complaints of eye irritation. Repeat MRI does demonstrate some growth of the tumor along the tympanic segment, but the patient is happy with her current results and will delay excision and cable grafting to a later date.

\section{DISCUSSION}

In the majority of situations, treatment of facial neuroma consists of tumor removal with cable nerve grafting..$^{2-8,10}$ Occasionally, the tumors may be able to removed without total nerve sacrifice, ${ }^{4}$ or at times the tumor is so large (case 2) that cable grafting is not feasible. Decompression is an alternative to resection in select cases,, 7 usually those patients with relatively good facial nerve function and small tumors who do not want the sequelae of total facial paralysis while hoping to recover to a grade III facial paresis, the best outcome one can expect after cable grafting. Resection and reanimation of facial neuromas requires the surgeon to be prepared to expose and treat the facial nerve from the CPA to the parotid branches. The surgeon must also have the ability to harvest the needed cable graft from either the great auricular or the sural nerve.

Stereotaxic radiation therapy (SRT) has recently been described in the treatment of acoustic neuromas. In searching the literature, we could not find any reports describing its use on facial neuromas. Our concern would be that facial reanimation is not addressed by SRT and diagnosis by way of biopsy is also not obtained. Patients who cannot medically tolerate a general anesthesia for a major surgical procedure may benefit from SRT in that it may retard the neuroma's growth. Other than this situation, SRT is probably not indicated to treat facial neuromas.

These three cases illustrate that the treatment parameters for facial nerve neuromas are not all clear cut. Treatment flexibility must be a part of one's armamentarium in the management of facial nerve neuromas.

\section{REFERENCES}

1. Schmidt C: Neurinom deo nervus facialis. Zentralbl Hals NasOhrenheilkd. 16:529, 1930 
2. Saleh E, Archilli V, Naguib M, et al.: Facial nerve neuromas: Diagnosis and Management. Am J Otol 16:521-526, 1995

3. Pillsbury HC, Price HC, Gardiner LJ: Primary tumors of the facial nerve: diagnosis and management. Laryngoscope 95: 1045-1048, 1983

4. McMenomey SO, Glasscock ME, Minor LB, Jackson CG, Strasnick B: Facial nerve neuromas presenting as acoustic tumors. Am J Otol 15:307-312, 1994

5. Pulec JL: Facial nerve neuroma. ENT J 73:721-752, 1994

6. Wiet RJ, Lotan AN, Monsell EM, Shambaugh GE: Tumor involvement of the facial nerve. Laryngoscope 93:1301-1309, 1983
7. O'Donoghue GM: Tumors of the facial nerve. In Brackmann DE, Jackler RK (eds). Neurotology. St. Louis, CV Mosby, 1994, pp 1321-1332

8. Gantz BJ, Weber PC: Idiopathic facial paralysis (Bell's palsy). In Rakel R (ed): Conn's Current Therapy, 1995. Philadelphia, WB Saunders, 1995, pp 869-871

9. House JW: Facial nerve grading system. Laryngoscope 93: 1056-1069, 1983

10. O'Donoghue GM, Brackmann DE, House JW, Jackler RK: Neuromas of the facial nerve. Am J Otol 10:49-54, 1989 